CS 2018.01.01.8

Bionatura Conference Series Vol 1. No 1. 2018

Publicación del IV CONGRESO INTERNACIONAL DE BIOTECNOLOGÍA Y BIODIVERSIDAD IV CIBB Y XV FORO INTERNACIONAL BANANERO

INVESTIGATION / RESEARCH

Previos / $\underline{\text { Index } / \text { Next }}$

\title{
Estudio fitoquímico, actividad antioxidante de especies de orquídeas de los géneros Epidemdrum, Oncidium y Caucaea
}

Phytochemical study, antioxidant activity of orchid species of the genera Epidemdrum, Oncidium and Caucaea

Marco Cerna ${ }^{1}$, Fernando Mencias $^{2}$, Telmo Salazar $^{3}$ Sara Gutiérrez $^{4}$

Disponible en: http://dx.doi.org/10.21931/RB/CS/2018.01.01.8

\section{RESUMEN}

En la presente investigación se tuvo como objetivo identificar metabolitos secundarios en extractos etanólicos de distintas especies de géneros de orquídeas mediante un screening fitoquímico, en donde se realizaron pruebas para alcaloides, flavonoides, saponinas, taninos y triterpenos. Además, se evaluó la capacidad antioxidante de 35 especies de orquídeas de los géneros Caucaea, Epidemdrum y Oncidium, para esto se utilizó el método DPPH para analizar la capacidad captadora de radicales libres, como control positivo para este análisis se utilizó vitamina $\mathrm{C}$.

Los resultados determinaron en el screening fitoquímico que los metabolitos secundarios que más presencia tuvieron en las especies estudiadas fueron flavonoides, triterpenos y saponinas. Las especies en estudio del género Caucaea y Oncidium presentaron reacción positiva para flavonoides y triterpenos en mayor cantidad, mientras que las muestras del género Epidendrum presentaron saponinas en un $100 \%$. 
En el análisis de actividad antioxidante con metodología DPPH, las especies más sobresalientes fueron Oncidium excavatum y Epidendrum nocturnun, la última especie se destacó por que necesitó una concentración de 3,50 ppm para inhibir el $50 \%$ de los radicales libres de DPPH presentes en una solución de ensayo.

Palabras claves: Antioxidante, orquídeas, DPPH, metabolitos, screening.

\section{ABSTRACT}

In the present investigation, the objective was to identify secondary metabolites in ethanolic extracts of different species of orchid genera through phytochemical screening, where tests were carried out for alkaloids, flavonoids, saponins, tannins and triterpenes. In addition, the antioxidant capacity of 35 species of orchids of the genera Caucaea, Epidemdrum and Oncidium was evaluated. For this, the DPPH method was used to analyze the free radical scavenging capacity. Vitamin C was used as a positive control for this analysis.

The results determined in the phytochemical screening that the secondary metabolites that had the most presence in the species studied were flavonoids, triterpenes and saponins. The species under study of the genus Caucaea and Oncidium presented positive reaction for flavonoids and triterpenes in greater quantity, while the sample of the genus Epidendrum presented saponins in $100 \%$.

In the analysis of antioxidant activity with DPPH methodology, the most outstanding species were Oncidium excavatum and Epidendrum nocturnun, the last species stood out because it needed a concentration of 3,50 ppm to inhibit 50 $\%$ of the free radicals of DPPH present in a solution of test

Keywords. Antioxidant, orchids, DPPH, metabolites, screening.

\section{INTRODUCCIÓN.}

Las orquídeas pertenecientes a la familia Orquidaceae, presentan flores muy llamativas debido a su gama amplia colores, formas y aromas; hasta el momento se han detallado alrededor de 28000 especies, y casi 900 géneros en sus diversas variantes ${ }^{1}$. Endara y Jost ${ }^{2}$ mencionan que existen 1707 especies de orquídeas endémicas en el Ecuador, por tal motivo Pitman et al ${ }^{3}$ dicen que esta familia es 
considerada la más diversa de las plantas vasculares.

Se estima que solo cerca del $6 \%$ de plantas superiores han sido estudiadas para comprobar su actividad biológica y de estas el $15 \%$ tiene un estudio fitoquímico; esto provoca el interés de la industria farmacéutica que ve el potencial de las plantas para la elaboración de nuevos medicamentos ${ }^{4}$.

Los metabolitos secundarios son sustancias complejas, usados como principios activos en la industria farmacéutica, estos presentan una acción farmacológica o fisiológica sobre el organismo, el valor económico de los metabolitos secundarios es muy alto a comparación con los metabolitos primarios ${ }^{5}$.

Los antioxidantes son sustancias que favorecen el retraso del daño a nivel celular causado por la acción oxidativa de los radicales libres ${ }^{6}$.

En la actualidad existe mucho interés en realizar estudios relacionados con los usos etnobotánicos de las plantas, o a su vez para obtener nuevos compuestos con actividad farmacológica, en especial compuestos antioxidantes. Debido a que Ecuador posee una gran diversidad de orquídeas ${ }^{7}$, que han sido utilizadas en medicina ancestral, se evidencia la falta de estudios científicos que corroboren dichas aplicaciones. Con lo antes mencionado es importante ya que se puede obtener principios activos que reemplacen a compuestos sintéticos utilizados actualmente en la industria farmacéutica ${ }^{8}$.

\section{MATERIALES Y MÉTODOS.}

\section{Recolección de la muestra.}

La recolección del material vegetal de los tres géneros: Caucaea, Epidemdrum y Oncidium; se realizó en la provincia de Pichincha, cuidad Quito, en la parroquia de El Quinche; en el "Orquideario de Sarina".

El material vegetal seleccionado para este estudio consistió en $10 \mathrm{~g}$ de hojas de 
cada especie, se cortaron las hojas con una tijera de podar previamente flameada y esterilizada con alcohol al $96 \%$, el material fue colocado en bolsas de papel y almacenadas en fundas plásticas con sierre para absorber la humedad se añadió silicagel a la funda.

\section{Obtención del extracto etanólico.}

El extracto se obtuvo mediante maceración de acuerdo a la metodología descrita por Moreno y Jaramillo ${ }^{9}$, el proceso consistió en: tomar $10 \mathrm{~g}$ de hojas jóvenes de cada una de las especies en estudio, se trituró en un mortero de porcelana de 80 mm de diámetro, se añadió $70 \mathrm{~mL}$ de etanol $96 \%$ y se maceró en un frasco ámbar durante 8 días en condiciones ambiéntales y completa oscuridad. Luego se tamizó con papel filtro para eliminar los residuos de hojas y se almacenó en la oscuridad en condiciones ambiéntales para evitar la degradación de las moléculas presentes en los extractos a analizar.

\section{Screening fitoquimico. Test para alcaloides.}

El ensayo se realizó de acuerdo a la metodología descrita por Carrera et al ${ }^{10}$, mediante el uso del reactivo de Draggendorf; para lo cual se colocó $2 \mathrm{~mL}$ de muestra en un tubo de ensayo, luego se agregó de 2 a 3 gotas del reactivo de draggendorf, se agitó fuertemente y se esperó por 30 minutos.

Se utilizó como control negativo alcohol al 96 \% y como control positivo cafeína.

Si se observa opalescencia $(+)$, turbidez $(++)$ o precipitado $(+++)$ se considera que la muestra contiene alcaloides.

\section{Determinación de flavonoides.}

El ensayo se realizó de acuerdo a la metodología descrita por Ramos et al ${ }^{11}$, mediante el uso del reactivo de Shinoda; para lo cual se colocó $2 \mathrm{~mL}$ de muestra en un tubo de ensayo, luego se agregó varias limaduras de magnesio, los tubos de ensayo se los colocó en un baño maría a $60{ }^{\circ} \mathrm{C}$, después se colocó 2 gotas de $\mathrm{HCl}$ 
concentrado.

\section{Test de saponinas.}

El ensayo se realizó de acuerdo a la metodología descrita por Moreno y Jaramillo 9 , mediante la prueba de espuma; para lo cual se colocó $2 \mathrm{~mL}$ de muestra en un tubo de ensayo, luego se agregó $5 \mathrm{~mL}$ de agua destilada y se agitó vigorosamente durante un minuto.

\section{Test de taninos.}

El ensayo se realizó de acuerdo a la metodología descrita por Bravo y Acuña ${ }^{12}$, mediante el uso del reactivo Gelatina-Sal; para lo cual se colocó $1 \mathrm{~mL}$ de muestra en un tubo de ensayo, luego se adicionó $1 \mathrm{~mL}$ de reactivo.

\section{Test de triterpenos.}

El ensayo se realizó de acuerdo a la metodología descrita por Carrera et al ${ }^{10}$, mediante el uso del reactivo de Liebermann-Burchard; para lo cual se colocó $1 \mathrm{~mL}$ de muestra en un tubo de ensayo, luego se adicionó lentamente por la pared del tubo $1 \mathrm{~mL}$ de anhídrido acético, finalmente con precaución se añadió 1-2 gotas de ácido sulfúrico concentrado.

\section{Evaluación de la actividad antioxidante.}

Para este ensayo se aplicó la técnica propuesta por Noriega et al ${ }^{13}$, para lo cual se elaboró una solución de 2,2-difenil-1-picrilhidracilo DPPH ( $1^{\prime} 10^{-4}$ en etanol), como control positivo se usó vitamina C. Para cada extracto se preparó diluciones en diferentes concentraciones: $10 \mathrm{uL}, 50 \mathrm{uL}$ y $80 \mathrm{uL}$ en viales ámbar, se completó con alcohol al $96 \%$ obteniendo un volumen final de $100 \mathrm{uL}$; a las diluciones realizadas se les agregó $2,9 \mathrm{~mL}$ de DPPH hasta completar el volumen final de 3 $\mathrm{mL}$ y se homogeniza, luego se almacena en la oscuridad durante 30 minutos. 
Las muestras se colocaron en celdas plásticas y se analizó tres veces en el espectrofotómetro de UV-visible (JASCO V-730) con el programa spectral manager, a $517 \mathrm{~nm}$ de longitud de onda. Previamente el espectrofotómetro fue encerado con alcohol al $96 \%$.

Las soluciones preparadas se colocaron en viales ámbar y a estas se les añadió 2,9 $\mathrm{mL}$ de DPPH preparado previamente, se agitó y almacenó en la oscuridad por 30 minutos.

Las soluciones preparadas se colocaron en celdas plásticas de $3 \mathrm{~mL}$ para ser analizadas en el espectrofotómetro (JASCO V-730) a una longitud de onda 517 $\mathrm{nm}$, se analizaron las muestras en orden creciente de su concentración, cada muestra se analizó por triplicado.

\section{RESULTADOS}

\section{Identificación de metabolitos secundarios}

Mediante las pruebas colorimétricas se identificó la presencia o ausencia de metabolitos secundarios como: alcaloides, flavonoides, saponinas, taninos y triterpenos en las orquídeas colectadas, usando la técnica cualitativa del Manual de Miranda y Cuellar ${ }^{14}$.

\section{Screening fitoquímico género Caucaea}

El porcentaje de presencia de metabolitos en las especies en estudio del género Caucaea fueron: flavonoides y triterpenos en un $100 \%$, saponinas en un $88 \%$, alcaloides y taninos con un 44,4\%; no existen otros estudios fitoquímicos del género Caucaea debido a que es un género nuevo que fue separado de Oncidium y se ha discutido mucho sobre la pertenencia de algunas especies del género Caucaea y Oncidium como lo señala Szlachetko y Kolanowska ${ }^{15}$. Sin embargo, en este trabajo se evidenció la presencia de los mismos grupos de metabolitos: flavonoides y triterpenos en los 2 géneros. 


\section{Screening fitoquímico del género Epidendrum}

El porcentaje de presencia de metabolitos en las especies en estudio del género Epidendrum fueron: saponinas $100 \%$, triterpenos $90,9 \%$, taninos $63,6 \%$, flavonoides $45,4 \%$ y alcaloides $0 \%$, ninguna muestra presentó alcaloides lo que contrasta con el estudio hecho por Sut et $\mathrm{al}^{16}$, que encontró alcaloides fenantrénicos en especies del género Epidendrum; esto indicaría que las especies colectadas no han tenido una amenaza externa que induzca la producción de alcaloides, como lo explica Farrán et al ${ }^{17}$.

\section{Screening fitoquímico del género Oncidium}

El porcentaje de presencia de metabolitos en las especies en estudio del género Oncidium fueron: flavonoides y triterpenos $100 \%$, saponinas $93,3 \%$, taninos 73,3 $\%$, alcaloides $66,6 \%$, Amar et $\mathrm{al}^{18}$ presentan un estudio realizado en Oncidium baueri, en el que también se encontró flavonoides y triterpenos. Particularmente en el presente estudio se encontró un alto contenido de flavonoides en las especies Oncidium excavatum y Oncidium sp1; factor indicador de actividad antioxidante, como lo señala González et al ${ }^{19}$. Ver tabla 1. 
Resultados metabolitos secundarios

\begin{tabular}{|c|c|c|c|c|c|c|c|}
\hline \multirow[t]{3}{*}{ Género } & \multirow[t]{3}{*}{ Especies } & \multicolumn{6}{|c|}{ Metabolitos secundarios } \\
\hline & & \multirow[t]{2}{*}{ Alcaloides } & \multirow[t]{2}{*}{ Flavonoides } & \multicolumn{2}{|c|}{ Saponinas } & \multirow[t]{2}{*}{ Taninos } & \multirow[t]{2}{*}{ Triterpenos } \\
\hline & & & & $1 \mathrm{~min}$ & $5 \mathrm{~min}$ & & \\
\hline \multirow{9}{*}{$\begin{array}{l}C \\
a \\
u \\
c \\
a \\
e \\
a\end{array}$} & Cancaea sp1 & - & + & + & - & - & + \\
\hline & Cancaea sp2 & - & + & ++ & ++ & ++ & ++ \\
\hline & Cancaea $\mathrm{sp} 3$ & - & +++ & + & + & ++ & +++ \\
\hline & Cancaea sp4 & + & ++ & ++ & ++ & ++ & + \\
\hline & Caucaea sp5 & - & ++ & + & + & ++ & ++ \\
\hline & Caucaea sp6 & + & ++ & - & - & - & + \\
\hline & Cancaea cucullata & - & ++ & + & + & - & + \\
\hline & Caucaea mubigena & + & ++ & ++ & ++ & - & + \\
\hline & Caucaea phalaenopsis & + & + & + & + & - & + \\
\hline \multirow{11}{*}{$\begin{array}{c}E \\
p \\
i \\
d \\
e \\
n \\
d \\
r \\
u \\
m\end{array}$} & Epidendrum blepharoclinium & - & - & ++ & ++ & + & + \\
\hline & $\begin{array}{l}\text { Epidendrum blepharoclinium } \\
\text { blanco }\end{array}$ & - & - & ++ & + & - & + \\
\hline & Epidendrum cochlidium sp1 & - & - & ++ & ++ & + & + \\
\hline & Epidendrum cochlidium $\mathrm{sp} 2$ & - & +++ & ++ & + & + & ++ \\
\hline & Epidendrum Jamiesonis & - & - & + & + & + & + \\
\hline & Epidendrum medusae & - & - & + & + & - & + \\
\hline & Epidendrum nocturnum & - & +++ & + & - & ++ & - \\
\hline & Epidendrum paniculatum & - & ++ & +++ & ++ & - & +++ \\
\hline & Epidendrum porphyreum & - & - & + & + & + & + \\
\hline & Epidendrum secundum & - & ++ & +++ & +++ & - & ++ \\
\hline & Epidendrum secundum blanco & - & + & +++ & +++ & ++ & + \\
\hline \multirow{15}{*}{$\begin{array}{c}O \\
n \\
c \\
i \\
d \\
i \\
u \\
m\end{array}$} & Oncidium $\mathrm{sp} 1$ & + & +++ & ++ & ++ & ++ & ++ \\
\hline & Oncidium sp2 & - & + & ++ & ++ & ++ & ++ \\
\hline & Oncidium $\mathrm{sp} 3$ & + & + & ++ & ++ & + & ++ \\
\hline & Oncidium sp4 & - & + & ++ & ++ & ++ & + \\
\hline & Oncidium $\mathrm{sp} 5$ & + & ++ & ++ & ++ & ++ & + \\
\hline & Oncidium $\mathrm{sp} 6$ & ++ & + & ++ & ++ & - & + \\
\hline & Oncidium sp 7 & - & ++ & +++ & +++ & ++ & + \\
\hline & Oncidium camilita portilla & ++ & ++ & + & + & - & ++ \\
\hline & Oncidium excavatum & + & +++ & +++ & +++ & ++ & + \\
\hline & Oncidium fuscatum & - & ++ & - & - & ++ & ++ \\
\hline & Oncidium obryzatum & - & + & +++ & +++ & + & + \\
\hline & Oncidium portmannii & ++ & + & + & + & - & + \\
\hline & Oncidium selecoamum & + & + & ++ & ++ & - & + \\
\hline & Oncidium strictum & + & ++ & + & + & + & + \\
\hline & Oncidium sweet sugar & + & ++ & + & + & ++ & + \\
\hline
\end{tabular}

Nota: Negativo (-), poca presencia $(+)$, moderada presencia $(++)$, alta presencia $(+++)$.

Elaborado por: (Los autores, 2018).

Tabla 1. Resultados metabolitos secundarios

Evaluación de la actividad antioxidante de los extractos 
Se observó que las especies analizadas de este género no tienen actividad antioxidante significativa ya que ninguna logra sobrepasar el $50 \%$ de inhibición, ver figura 1.

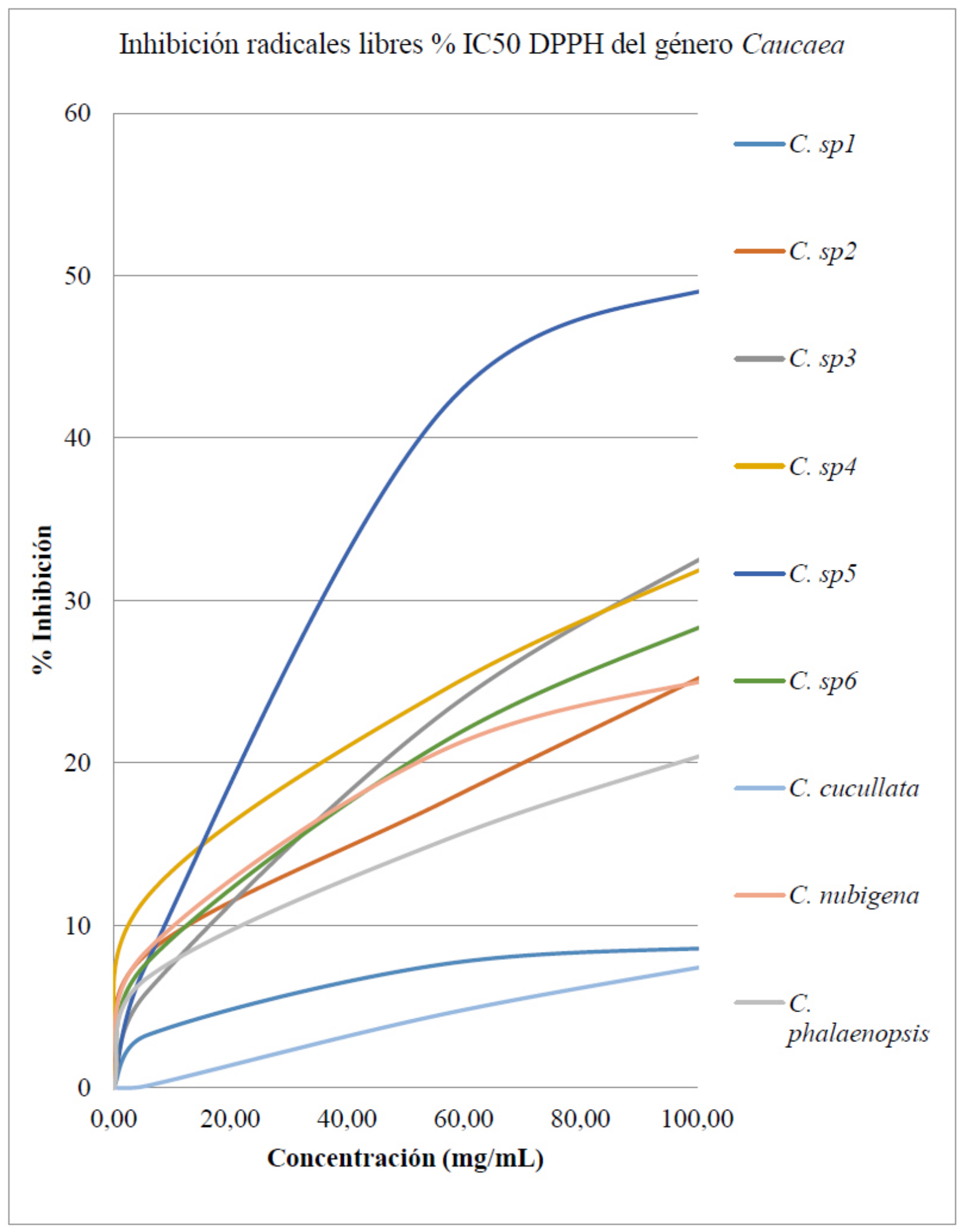

Nota: Curvas de porcentajes de inhibición de radicales libres obtenidas mediante Microsoft Excel 
Figura 1. Porcentaje de inhibición del DPPH del género Caucaea

Actividad antioxidante del género Epidendrum

Entre las especies analizadas se destaca E. nocturnum que presentó una capacidad antioxidante del $50 \%$ de los radicales DPPH con una concentración de 3,50 ppm, las demás especies tienen una actividad antioxidante muy baja, ver figura 2 . 


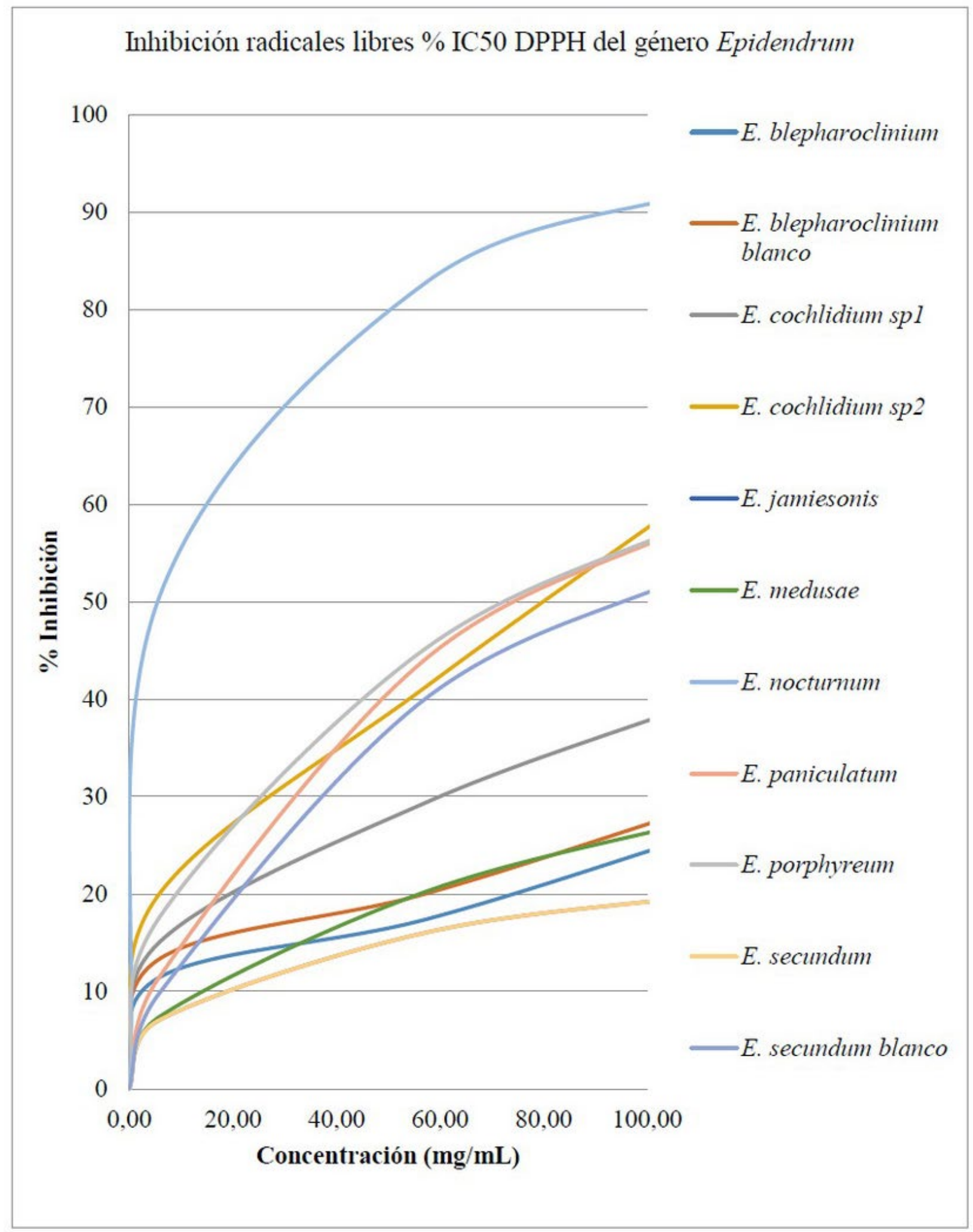

Nota: Curvas de porcentajes de inhibición de radicales libres obtenidas mediante Microsoft Excel 2013.

Figura 2. Porcentaje de inhibición del DPPH del género Epidendrum 
Se puede observar que destacan 2 especies por su potencial antioxidante las cuales son: $O$. sp7 y $O$. excavatum, las cuales a una concentración de 43 y $31 \mathrm{ppm}$ respectivamente, reducen el $50 \%$ de los radicales DPPH; las otras especies no tienen una actividad antioxidante significativa, ver figura 3.

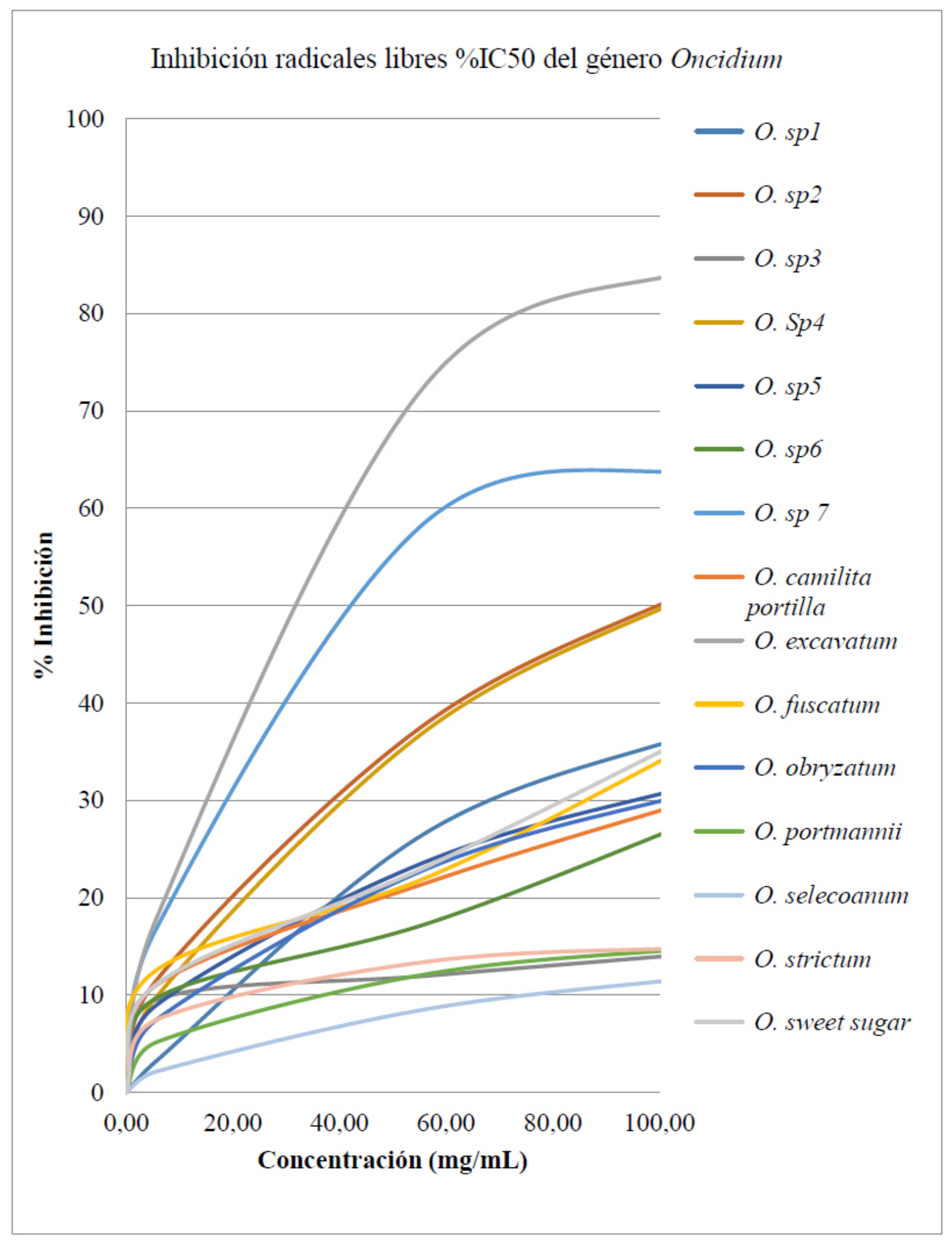


Nota: Curvas de porcentajes de inhibición de radicales libres obtenidas mediante Microsoft Excel 2013.

Figura 3. Porcentaje de inhibición del DPPH del género Oncidium

\section{Análisis estadístico de la actividad antioxidante}

Se realizó un análisis estadístico por conglomerados, el cual se dividió en 3 clústers: poca actividad, moderada actividad y alta actividad. De color rojo el conglomerado 1 nos muestra las especies con poca actividad antioxidante; de color verde el conglomerado 2 muestra especies que tienen una actividad antioxidante moderada y por ultimo de color azul el conglomerado 3 nos muestra especies que tienen una alta actividad antioxidante, ver figura 4.

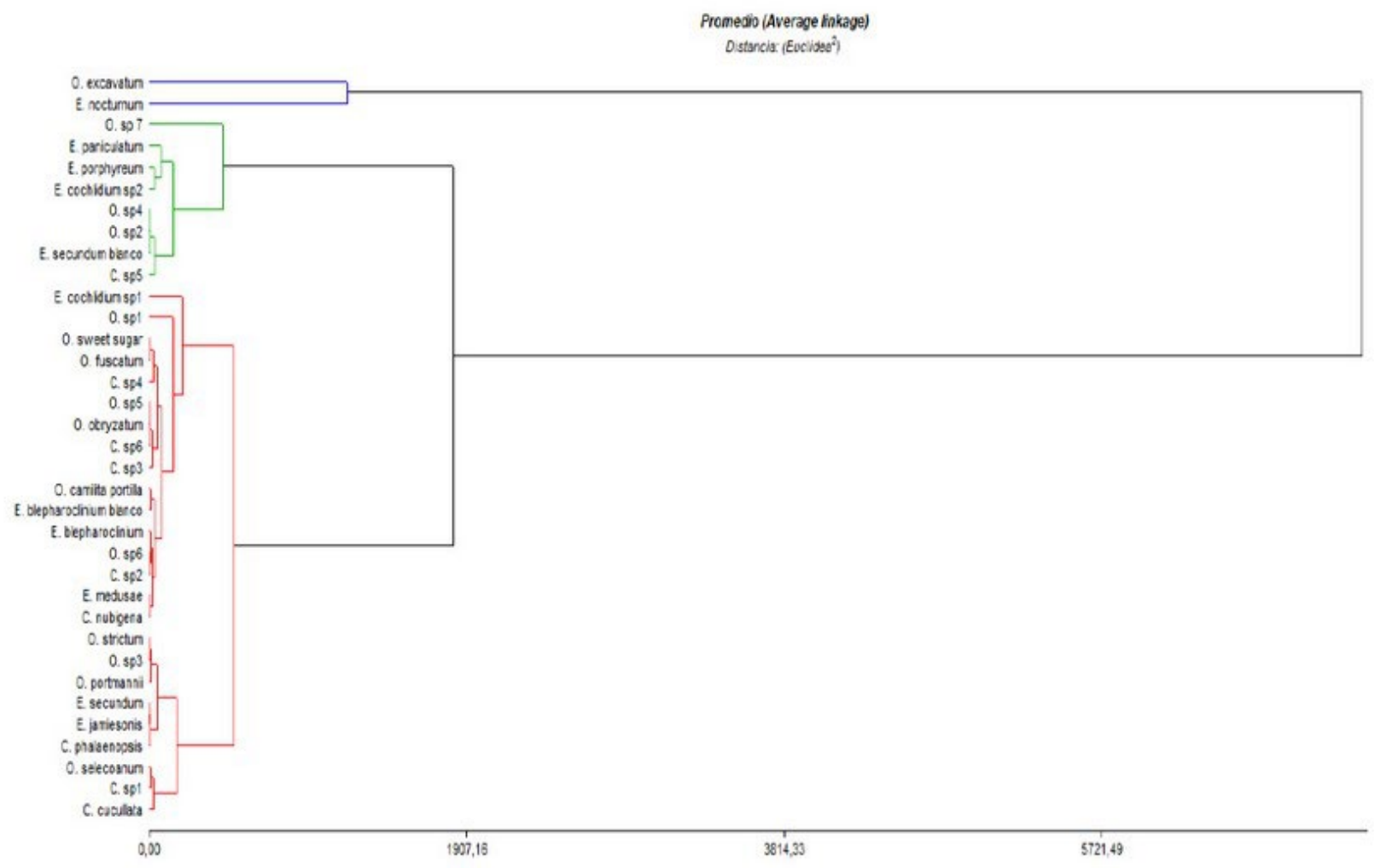

Nota: Obtenidas mediante Infostat 2018.

Figura 4. Cuadro de clasificación de especies mediante análisis de conglomerados.

El clúster 3 está compuesto de las especies Epidendrum nocturnum y Oncidium 
excavatum; las cuales redujeron el $50 \%$ de los radicales libres de DPPH con tan solo 3,5 y $31 \mathrm{ppm}$ respectivamente lo cual las cataloga como especies con alta actividad antioxidante respecto a las otras muestras.

Se pudo observar que la especie Epidendrum nocturnum superó la capacidad antioxidante de Prosthechea michuacana reportada por González et al ${ }^{19}$ en su estudio "Aislamiento e identificación de los compuestos con actividad antioxidante de la orquídea comestible Prosthechea michuacana", que requirió 13,22 ppm para inhibir el \% IC50 de DPPH.

Según señala González et al ${ }^{19}$ compuestos como los flavonoides infiere en el potencial antioxidante de las plantas, lo que confirman los resultados de este ensayo, ya que en el screening fitoquímico de las 2 especies con alta actividad antioxidante presentaron un alto contenido de flavonoides en las pruebas colorimétricas que se realizaron.

\section{CONCLUSIONES}

Los metabolitos secundarios encontrados en las especies estudiadas fueron: triterpenos 96,96\%, saponinas 96,65\%, flavonoides $81,8 \%$, taninos $60,43 \%$ y alcaloides $37 \%$.

Las especies estudiadas del género Caucaea y Oncidium mostraron una estrecha similitud al presentar 2 metabolitos en las especies analizadas, los cuales fueron: flavonoides y triterpenos en un $100 \%$ de las especies.

El análisis de actividad antioxidante señaló a 2 especies como las de mayor acción, las cuales fueron Epidendrum nocturnum con un \% IC50 de 3,5 ppm y Oncidium excavatum con un \% IC50 de $31 \mathrm{ppm}$.

Las 2 especies que presentaron una capacidad antioxidante significativa mostraron una presencia alta de flavonoides en su composición, lo que confirma que este metabolito secundario aporta a la capacidad antioxidante de estas plantas. 


\section{REFERENCIAS}

1. The Plant List. The Plant List: A working list of all plants species [Internet]. 2013 [cited 2018 Feb 2]. Available from:

http://www.theplantlist.org/1.1/browse/A/Orchidaceae/

2. Endara L, Jost L. Orchidaceae. In: León-yánez S, Valencia R, Pitman N, Endara L, Ulloa C, Navarrete H, editors. Libro rojo de las plantas endémicas del Ecuador. Segunda. Quito: Herbario QCA, Pontificia Universidad Católica del Ecuador; 2011. p. 80.

3. Pitman N, Valencia R, León-Yánez S. El libro rojo de las plantas endémicas del Ecuador. Segunda. Quito: Herbario QCA, Pontificia Universidad Católica del Ecuador; 2012. 80-85 p.

4. Patiño N. Farmacología médica. México D.F.: Médica Panamericana; 2008. 921-928 p.

5. Cano A, Arnao M. Hydrophilic and lipophilic antioxidant activity in different leaves of three lettuce varieties. Int J Food Prop. 2005;8(3):521-8.

6. Youngson R. Antioxidantes y radicales libres. Madrid: EDAF; 2003. 112$115 \mathrm{p}$.

7. Endara L, Williams N, León-Yánez S. 1. Explorando los patrones de endemismo de las orquídeas ecuatorianas: Implicaciones para su conservación. In: X congreso Latinoamericano de Botánica. La Serena; 2010.

8. Rivas-Morales C, Oranday-Cárdenas M, Verde-Star M. Investigación en plantas de importancia médica. Nuevo León: OmniaScience; 2016. 105-121 p.

9. Moreno N, Jaramillo B. Análisis fitoquímico preliminar de Pachira quinata (Jacq.) W.S. Alverson, Bogotá, Colombia. Boletín Semillas Ambient. 2017;11(1):30-9.

10. Carrera G, Benedito E, Souza-Leal T, Pedroso-De-Moraes C, Gaspi F. Testes fitoquímicos em extratos foliares de Oeceoclades maculata Lindl. (Orchidaceae). Rev Bras Plantas Med. 2014;16(4):938-44. 
11. Ramos P, Colareda G, Rosella M, Debenedetti S, Spegazzini E, Consolini A. Phytochemical profile and anti-inflammatory effect of the orchid Catasetum macroglossum. Lat Am J Pharm. 2012;31(1):62-7.

12. Bravo A, Acuña WD. Evaluación fitoquímica y determinación de flavonoides en hojas de Ficus benjamina L. Xilema. 2015;28(1):61-7.

13. Noriega P, Mosquera T, Baldisserotto A, Abad J, Aillon C, Cabezas D, et al. Chemical Composition and Biological Activities of the Essential Oil from Leaves of Cleidion javanicum Bl. J Essent Oil Bear Plants [Internet]. 2012;15(2):29-31. Available from: http://www.tandfonline.com/doi/abs/10.1080/0972060X.2012.10644035

14. Miranda M, Cuellar A. Manual de Prácticas de Laboratorio: Farmacognosia y Productos Naturales. Primera. La Habana: Universidad de la Habana; 2000. 34$50 \mathrm{p}$.

15. Szlachetko D, Kolanowska M. Five new species of Caucaea (orchidaceae) from Colombia and Ecuador. Polish Bot J. 2015;60(2):127-34.

16. Sut S, Maggi F, Dall'Acqua S. Bioactive secondary metabolites from orchids (Orchidaceae). Chemistry \& biodiversity, 2017. Chem Biodivers. 2017;14(11):1-30.

17. Farrán A, López C, Pérez M, Santa María M. Química bioorgánica y productos naturales. Segunda. Claramunt R, editor. Madrid: UNED; 2017. 315$321 \mathrm{p}$.

18. Amar M, Baba S, Singh A, Singh J, Sembi J, Arora M, et al. A review on phytochemical and pharmacological potential of family orchidaceae. Artic Int Res J Pharm Mamta Arora al Int Res J Pharm. 2017;8(10):9-24.

19. González A. Aislamiento e identificación de los compuestos con actividad antioxidante del extracto de cloroformo de la orquídea comestible Prosthechea michuacana. Instituto Politécnico Nacional; 2009.

Marco Cerna ${ }^{1}$, Fernando Mencias $^{2}$, Telmo Salazar $^{3}$ Sara Gutiérrez ${ }^{4}$

1 Grupo de investigación Nunkui Wakan, Universidad politécnica Salesiana del 
Ecuador, Carrera de Biotecnología mcerna@ups.edu.ec ORCID ID https://orcid.org/0000-0002-0911-9900

2 Grupo de investigación Nunkui Wakan, Universidad politécnica Salesiana del Ecuador, Carrera de Biotecnología sdqfernando90@hotmail.com ORCIDID https://orcid.org/0000-0001-7495-2053

3 Grupo de investigación Nunkui Wakan, Universidad politécnica Salesiana del Ecuador, Carrera de Biotecnología telmofsp@hotmail.com ORCIDID https://orcid.org/0000-0002-2109-5243

4 Jardín Botánico "Orquídeas de Sarina” el Quinche, Quito - Ecuador, atrujillo166@hotmail.com 